\title{
Perioperative anesthetic management in pediatric hereditary angioedema; case report
}

\author{
M. Nuri Deniz, Aylin Incesu, Elvan Erhan, Gulden Ugur
}

Ege University Medical Faculty, İzmir, Turkey

Email: mnurideniz@,hotmail.com

Received 26 July 2012; revised 24 October 2012; accepted 30 October 2012

\begin{abstract}
Hereditary angioedema is a rare but life-threatening disease, usually resulting from upper respiratory tract traumas and stress. In this case report, we present the management of a 14-year-old female patient who was diagnosed with hereditary angioedema and scheduled to undergo transurethral resection of bladder (TURB) procedure for bladder tumor. She was on prophylactic danazol treatment and prior to the operation the dose of danazol was increased. On the day of the operation, patient was given C1-IHN concentrate and was sedated. In conclusion, hereditary angioedema is a rare disease in which multidisciplinary and aggressive approach during anesthesia would yield successful results.
\end{abstract}

Keywords: Hereditary Angioedema; C1 Inhibitor Deficiency; Anesthetic Management and Bladder Tumor

\section{INTRODUCTION}

C1 Inhibitor (C1 INH) deficiency is an autosomal dominant disorder that causes hereditary angioedema (HAE) [1]. C1 INH deficiency can result in mortality by causing sudden bouts of serious angioedema [2]. Local traumas to the upper respiratory tract during operations (such as dental treatments, tonsillectomy and endotracheal intubation) pose serious risk for HAE attacks [3]. Therefore, care should be taken with regard to preoperative preparations and the technique of anesthesia in patients diagnosed with HAE and scheduled for operation. In this case report, we presented the perioperative anesthetic management of the patient with HAE who was scheduled for transurethral resection of bladder tumor (TURBT).

\section{CASE REPORT}

A 14-year-old female patient (141 cm height, $52 \mathrm{~kg}$ weight) was scheduled for TURBT. Pre-operative examination revealed normal hematological and biochemical tests and physical examination. History of the patient showed that she had been using propranolol HCI $40 \mathrm{mg} /$ day for sinus tachycardia until 6 months earlier. The patient had her first HAE attack when she was 11 years old. Edema in her hands and feet occurred after she played volleyball. HAE attacks continued and presented as edema in lower lips, perineum and peripheral limb. During one year she admitted to different pediatrics and finally she was diagnosed as HAE at the specialized children hospital, a disorder also identified in her father and her sister. The family history also revealed that her grandfather died suddenly following symptoms suggesting HAE. She was first treated with tranexamic acid. However her HAE attacks did not respond to tranexamic acid treatment. Therefore, danazol capsule $100 \mathrm{mg} /$ day BID was tried and found successful, which have been given by her pediatric immunology specialist. She was under control with no episodes of angioedema.

The patient has been under control for $\mathrm{C} 1 \mathrm{INH}$ deficiency and has been receiving prophylactic Danazol capsule $100 \mathrm{mg} /$ day BID, which have been given by her pediatric immunology specialist. She was generally healthy and no other medical conditions necessitating treatment were present. The dose of Danazol capsule was increased to $200 \mathrm{mg}$ /day BID 5 days before the operation. Forty five minutes before the operation, intravenous (IV) access was established and the patient was given $250 \mathrm{U}$ of purified C1-IHN concentrate (Berinert ${ }^{\circledR} \mathrm{P}$, CSL Behring, Germany), followed by IV midazolam $0.03 \mathrm{mg} \cdot \mathrm{kg}^{-1}$. All the resuscitative measures were prepared in the operating room. Ear-nose-throat specialist was ready for a possible emergency tracheostomy. The patient was taken into the operating room and she was monitored with electrocardiogram (ECG), Blood Pressure (BP), Heart Rate (HR) and $\mathrm{SpO}_{2}$. Following pre-oxygenation, anesthesia was induced with atropine $0.5 \mathrm{mg} \mathrm{IV}$, propofol $2 \mathrm{mg} \cdot \mathrm{kg}^{-1}$ IV, fentanyl $0.05 \mathrm{mg}$ IV and rocuronium $0.6 \mathrm{mg} \cdot \mathrm{kg}^{-1}$. Once sufficient muscle relaxation was achieved, the patient was intubated by $6-\mathrm{mm}$ endotracheal tube and anesthesia was maintained by $\mathrm{O}_{2}$, air and sevoflurane ( $2 \%$ $2.5 \%$ ). The patient's vital parameters were within normal limits throughout the surgery, which lasted 40 minutes 
(min). At the end of the operation the patient was given paracetamol $1000 \mathrm{mg}$ IV and tramadol $50 \mathrm{mg}$ IV over a period of 20 minutes for postoperative analgesia. Muscle relaxation was reversed with atropine $0.5 \mathrm{mg} \mathrm{IV}$ and neostigmine $1.0 \mathrm{mg}$ IV. The patient was extubated before being awake and she was ventilated via mask until sufficient respiration was restored. The patient was taken into the postoperative care unit and followed-up for 24 hours and discharged without any problems. The patient used danazol $200 \mathrm{mg} /$ day BID in the first two postoperative days. She continued danazol $100 \mathrm{mg} /$ day BID afterwards for prophylaxis.

\section{DISCUSSION}

C1-INH is the major regulator of early activation stage of classical complement pathway [4]. Immune complexes trigger the activation of $\mathrm{C} 1$, the first complement to $\mathrm{C} 1$ esterase. Activation of this pathway induces formation of $\mathrm{C} 2,4$ (C3) complex and C2, 4 causes production of anaphylactic, chemotactic and vasoactive peptides. C1-INH deficiency causes inappropriate or premature activation of $\mathrm{C} 1$ to $\mathrm{C} 1$ esterase and $\mathrm{C} 1$ esterase activity resumes unchecked [5]. This, in turn, results in episodic, subcutaneous and submucosal edema of the upper respiratory tract and gastrointestinal system [6]. Edema of the upper respiratory tract results in life-threatening airway obstruction [7]. Diagnosis of C1-INH deficiency is established by clinical findings, family history, measurement of complement levels and molecular genetic tests [8]. Trauma, emotional stress, surgical or diagnostic procedures in the cranial or cervical region, physiological changes in sex hormones (puberty, menstrual cycle or pregnancy), changes in climate, certain food and medical products can trigger angioedema [1]. In the present case, we noted during preanesthetic evaluation that the patient and her family had been diagnosed with HAE. Management of C1-INH deficiency includes prevention of angioedema as well as control of acute attacks [8]. Antifibronolytic agents (tranexamic acid) or epsilon-amino-caproic acid or synthetic weak androgens (danazol, stanozolol and oxandrolon) can be used for long-term prophylaxis [9]. For short-term prophylaxis, on the other hand, C1-INH concentrate, synthetic weak androgens and tranexamic acid are used [9]. Tranexamic acid or weak androgens are given to patients 5 days before an operation or dental procedure and they are continued until 2 days after the procedures [10]. When there is an acute attack, C1-INH concentrate, synthetic weak androgens and tranexamic acid should be used concomitantly. Fresh frozen plasma can be used instead if there is no $\mathrm{C} 1$-INH concentrate available [9]. Since the recommended doses of danazol and C1-INH concentrate for short term prophylaxis are $100-600 \mathrm{mg}$ and 500 - $1500 \mathrm{U}$ (effective for 24 - 48 hours), respectively, danazol dose, which our patient was using for long term prophylaxis, was doubled and C1-INH concentrate was injected IV $45 \mathrm{~min}$. before the operation. Premedication with midazolam was initiated to prevent emotional stress. In order to minimize the trauma, following induction, orotracheal intubation was performed once full curarization was achieved. Paracetamol and tramdol were injected for postoperative analgesia before the patient was awake and the patient was extubated before decurarization.

Hereditary angioedema most often presents in childhood. Almost $40 \%$ had onset of symptoms before the age of 5 years and $75 \%$ before the age 15 , occasional patients will have their first symptoms even earlier [9]. Identification, avoidance and elimination of precipitating factors that may trigger acute HAE attacks are crucial to prevent life-threatening complications [11]. In order of decreasing prevalence, the most common triggering factors identified in the pediatric population are: mechanical trauma $(52.6 \%)$, followed by mental stress $(36.8 \%)$, air-way infection $(36.8 \%)$, and menses $(26.7 \%)$ [11,12]. Therefore in pediatric population surgery, which includes endotracheal intubation (trauma to the upper respiratory tract) and surgical trauma as well psychogenic stress is an important factor that may trigger HAE attacks. In general, short-term prophylaxis to prevent acute angioedema exacerbation after surgical procedure and intubation consist of C1-IHN concentrate [11]. The traditional approach to long-term prophylaxis involves use of attenuated androgens and antifibrinolytics [11]. However long term prophlaxis with the use of antifibrinolytics and androgens are not recommended because of the serious side effects of these drugs in children. Attenuated androgens are typically not recommended for use in children because of obvious concerns over growth and development. In addition, the potential adverse effects associated with attenuated androgens are both numerous and concerning, ranging from weight gain to organ damage and tumor development $[11,13,14]$. Their efficacy may decline over time with use, despite an increase in dose [11,12]. Antifibrinolytics are frequently mentioned in older HAE protocols $[11,13,14]$. It has been shown that patients using antifibrinolytics for prophylaxis had a very modest reduction in frequency and duration of attacks in compareson to patients who used no prophylaxis at all $[11,15]$. For children antifibrinolytics are preferred to androgens [9]. In our case, the patient was first treated with tranexamic acid, but her HAE attacks did not respond to tranexamic acid. Therefore danazol was tried and found successful since she was under control with no episodes of angioedema. Newer safer and more effective alternatives for $\mathrm{HAE}$ are being developed but no experience is available yet with pediatric use [11].

Shah and Jacobs [16] reported their ten years' experience in pediatric angioedema. In their series of 10 chil- 
dren, they reported that one child, who underwent dental surgery under general anesthesia, was prophylactically treated with fresh frozen plasma and danazol. She experienced no perioperative angioedema and was discharged from the hospital to home the same day. Recently Yazawa et al. [17] reported the prophylactic administration of C1-INH concentrate in an emergency laparotomy to a pediatric patient ( 8 years old) who was suspected of having HAE because of the family history. Considering the high mortality rate resulting from laryngeal edema triggered by intubation, the patient's mother and maternal grandmother had been diagnosed with HAE. They decided to treat the patient as an HAE suspect because of the family history and the autosomal dominant form of interitance. They administered $500 \mathrm{U}$ C1-INH concentrate prophylactically before the surgery, they also repeated $500 \mathrm{U}$ of $\mathrm{C} 1-\mathrm{INH}$ concentrate in the postoperative pediatric ward. However, the authors did not report any information about premedication for anxiolysis and postoperative pain therapy. In our case, we gave special attention for sedation preoperatively and postoperative pain control since stress can also trigger angioedema [1].

Preoperative assessment is crucial to prevent life-threating complications associated with HAE. Clinical symptoms, family history and laboratory studies of complement and C1-INH are all important for the diagnosis of HAE. Clinical presentations in combination with a positive family history should raise suspicions regarding HAE [15]. However, $25 \%$ of HAE is the result of spontaneous mutation and a novel mutation may explain cases of angioedema with no family history $[11,18]$.

HAE is a rare disorder, and the attacks are seen during childhood in most patients. Since HAE may also result from spontaneous mutation, detailed history taking is crucial to recognize the symptoms suggesting HAE. With awareness of the possibility of HAE in pediatric population, appropriate perioperative management can be planned including minimization of trauma and emotional stress as well as prophylactic measures.

It was the first time that a pediatric patient diagnosed with HAE was operated for bladder tumor in our urology department. Surgery was a success with appropriate evaluation and anesthetic management. We presented this case to emphasize the importance of a careful history during pre-anesthesia assessment. We concluded that a multidisciplinary and aggressive anesthesiological approach yields successful results in a patient diagnosed with hereditary angioedema.

\section{REFERENCES}

[1] Agostoni, A., Aygoren-Pursun, E., Binkley, K.E., Blanch, A., Bork, K., Bouillet, L., Bucher, C., et al. (2004) Here- ditary and acquired angioedema: Problems and progress: Proceedings of the 3rd C1 esterase inhibitor deficiency workshop and beyond. Journal of Allergy and Clinical Immunology, 114, 51-131. doi:10.1016/j.jaci.2004.06.047

[2] Bruce, L.Z. (2010) The pathophysiology of hereditary angioedema. WAO Journal, 3, 25-28. doi:10.1097/WOX.0b013e3181f3f21c

[3] Bork, K. and Barnstedt, S.E. (2003) Laryngeal edema and death from asphyxiation after tooth extraction in four patients with hereditary angioedema. The Journal of the American Dental Association, 134, 1088-1094.

[4] Johnson, A.M., Alper, C.A., Rosen, F.S. and Craig, J.M. (1971) C1 inhibitor: Evidence for decreased hepatic synthesis in hereditary angioneurotic edema. Science, 173, 553-554. doi:10.1126/science.173.3996.553

[5] Fay, A. and Abinun, M. (2002) Current management of hereditary angioedema (C'1 esterase inhibitor deficiency). Journal of Clinical Pathology, 55, 266-270. doi:10.1136/jcp.55.4.266

[6] Nathani, F., Sullivan, H. and Churchill, D. (2006) Pregnancy and $\mathrm{C} 1$ esterase inhibitor deficiency: A successful outcome. Archives of Gynecology and Obstetrics, 274, 381-384. doi:10.1007/s00404-006-0183-6

[7] Zuraw, B.L. (2008) Hereditary angioedema. The New England Journal of Medicine, 359, 1027-1036. doi:10.1056/NEJMcp0803977

[8] Barakat, J. (2010) Hereditary angioedema in children. Angioedema, 1, 15-19.

[9] Gompels, M.M., Lock, R.J., Abinun, M., Bethune, C.A., Davies, G., Grattan, C., et al. (2005) C1 inhibitor deficiency: Consensus document. Clinical \& Experimental Immunology, 139, 379-394. doi:10.1111/j.1365-2249.2005.02726.x

[10] Agostoni, A., Cicardi, M., Cugno, M. and Storti, E. (1993) Clinical problems in the $\mathrm{C} 1$-inhibitor deficient patient. Behring Institute Mitteilungen, 93, 306-312.

[11] Hsu, D. and Shaker, M. (2012) An update on hereditary angioedema. Current Opinion in Pediatrics, 24, 638-646. doi:10.1097/MOP.0b013e328357b25e

[12] Farkas, H. (2010) Pediatric hereditary angioedema due to C1-inhibitor deficiency. Allergy, Asthma \& Clinical Immunology, 6, 18. doi:10.1186/1710-1492-6-18

[13] Frank, M.M. (2011) Update on preventive therapy (prophylaxis) of hereditary angioedema. Allergy and Asthma Proceedings, 32, 17-21. doi:10.2500/aap.2011.32.3412

[14] Maurer, M. and Magerl, M. (2011) Long-term prophylaxis of hereditary angioedema with androgen derivates: A critical appraisal and potential alternatives. Journal der Deutschen Dermatologischen Gesellschaft, 9, 99-107. doi:10.1111/j.1610-0387.2010.07546.x

[15] Zanichelli, A., Vacchini, R., Badini, M., Penna, V. and Cicardi, M. (2011) Standard care impact on angioedema because of hereditary $\mathrm{C} 1$ inhibitor deficiency: A 21month prospective study in a cohort of 103 patients. Allergy, 66, 192-196. doi:10.1111/j.1398-9995.2010.02433.x

[16] Udayan, K.S. and Jacobs, I.N. (1999) Pediatric angioe- 
dema. Archives of Otolaryngology-Head \& Neck Surgery, 125, 791-795.

[17] Yazawa, T., O’higashi, T., Daijo, H. and Shinomura, T. (2010) Anesthesia management for emergency laparotomy in a pediatric patient with suspected hereditary angioedema. Journal of Anesthesia, 24, 121-123.

doi:10.1007/s00540-009-0832-6
[18] Malik, S.S., Uppal, H., Sinha, A., Katam, K. and Srinivasan, K. (2011) Acute paediatric compartment syndrome of the hand caused by hereditary angioedema. Archive of Annals of the Royal College of Surgeons of England, 93, 138-140. doi:10.1308/147870811X602159 